Supplement of J. Micropalaeontol., 37, 403-429, 2018

https://doi.org/10.5194/jm-37-403-2018-supplement

(C) Author(s) 2018. This work is distributed under

the Creative Commons Attribution 4.0 License.

(c) (1)

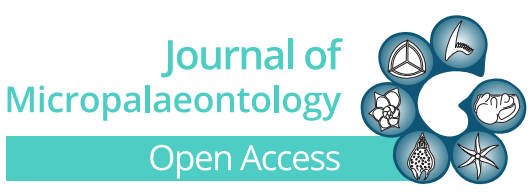

Supplement of

\title{
Assessing proxy signatures of temperature, salinity, and hypoxia in the Baltic Sea through foraminifera-based geochemistry and faunal assem- blages
}

Jeroen Groeneveld et al.

Correspondence to: Jeroen Groeneveld (jgroeneveld@uni-bremen.de)

The copyright of individual parts of the supplement might differ from the CC BY 4.0 License. 
Table S1) Summary of absolute counts of live (stained) and dead foraminifer assemblages at Anholt and Hanö Bay. At C-Ha-1 replicate core tops were collected, noted as $1 \mathrm{a}$ and $1 \mathrm{~b}$.

\begin{tabular}{|l|c|c|c|c|c|c|c|c|c|}
\hline Morphospecies (\%) & $\begin{array}{c}\text { C-An- } \\
\text { 1a Live }\end{array}$ & $\begin{array}{c}\text { C-An-1a } \\
\text { Dead }\end{array}$ & $\begin{array}{c}\text { C-An-1a } \\
\text { Total }\end{array}$ & $\begin{array}{c}\text { C-Ha-1a } \\
\text { Live }\end{array}$ & $\begin{array}{c}\text { C-Ha-1a- } \\
\text { Dead }\end{array}$ & $\begin{array}{c}\text { C-Ha-1a } \\
\text { Total }\end{array}$ & $\begin{array}{c}\text { C-Ha-1b } \\
\text { Live }\end{array}$ & $\begin{array}{c}\text { C-Ha-1b } \\
\text { Dead }\end{array}$ & $\begin{array}{c}\text { C-Ha-1b } \\
\text { Total }\end{array}$ \\
\hline Ammonia sp. (T6) & 0 & 0 & 0 & 43 & 1 & 44 & 37 & 0 & 37 \\
\hline Ammonia batava & 0 & 2 & 2 & 0 & 0 & 0 & 0 & 0 & 0 \\
\hline Ammotium cassis & 0 & 2 & 2 & 0 & 0 & 0 & 0 & 0 & 0 \\
\hline $\begin{array}{l}\text { Ammonia } \\
\text { falsobeccarii }\end{array}$ & 3 & 12 & 15 & 0 & 0 & 0 & 0 & 0 & 0 \\
\hline $\begin{array}{l}\text { Adercotryma } \\
\text { glomerata }\end{array}$ & 0 & 7 & 7 & 0 & 0 & 0 & 0 & 0 & 0 \\
\hline $\begin{array}{l}\text { Ammoscalaria } \\
\text { pseudospiralis }\end{array}$ & 4 & 4 & 8 & 0 & 0 & 0 & 0 & 0 & 0 \\
\hline Ammodiscus sp. & 0 & 1 & 1 & 0 & 0 & 0 & 0 & 0 & 0 \\
\hline $\begin{array}{l}\text { Bulimina } \\
\text { marginata }\end{array}$ & 27 & 117 & 144 & 0 & 0 & 0 & 0 & 0 & 0 \\
\hline $\begin{array}{l}\text { Bolivina } \\
\text { pseudopunctata }\end{array}$ & 0 & 0 & 0 & 0 & 0 & 0 & 0 & 0 & 0 \\
\hline $\begin{array}{l}\text { Cribrostomoides } \\
\text { jeffreysi }\end{array}$ & 1 & 3 & 4 & 0 & 0 & 0 & 0 & 0 & 0 \\
\hline $\begin{array}{l}\text { Cassidulina } \\
\text { laevigata }\end{array}$ & 35 & 0 & 35 & 0 & 0 & 0 & 0 & 0 & 0 \\
\hline $\begin{array}{l}\text { Cibicidoides } \\
\text { lobatulus }\end{array}$ & 0 & 3 & 3 & 0 & 0 & 0 & 0 & 0 & 0 \\
\hline $\begin{array}{l}\text { Cibicides/Cibicidoid } \\
\text { es spp. }\end{array}$ & 0 & 1 & 1 & 0 & 0 & 0 & 0 & 0 & 0 \\
\hline $\begin{array}{l}\text { E. clavatum- } \\
\text { selseyense complex }\end{array}$ & 1 & 48 & 49 & 273 & 236 & 509 & 300 & 106 & 406 \\
\hline $\begin{array}{l}\text { Eggerelloides } \\
\text { medius/scaber }\end{array}$ & 12 & 41 & 53 & 0 & 0 & 0 & 0 & 0 & 0 \\
\hline Lagena sp. & 0 & 1 & 1 & 0 & 0 & 0 & 0 & 0 & 0 \\
\hline $\begin{array}{l}\text { Lagenammina } \\
\text { difflugiformis }\end{array}$ & 0 & 9 & 9 & 0 & 0 & 0 & 0 & 0 & 0 \\
\hline Leptohalysis scotti & 9 & 1 & 10 & 0 & 0 & 0 & 0 & 0 & 0 \\
\hline
\end{tabular}




\begin{tabular}{|c|c|c|c|c|c|c|c|c|c|}
\hline $\begin{array}{l}\text { Miliolinella } \\
\text { subrotunda }\end{array}$ & 8 & 1 & 9 & 0 & 0 & 0 & 0 & 0 & 0 \\
\hline $\begin{array}{l}\text { Nonionella aff. } \\
\text { stella }\end{array}$ & 44 & 0 & 44 & 0 & 0 & 0 & 0 & 0 & 0 \\
\hline $\begin{array}{l}\text { Nonionellina } \\
\text { labradorica }\end{array}$ & 15 & 16 & 31 & 0 & 0 & 0 & 0 & 0 & 0 \\
\hline Nonionella turgida & 56 & 0 & 56 & 0 & 0 & 0 & 0 & 0 & 0 \\
\hline Pyrgo williamsoni & 2 & 0 & 2 & 0 & 0 & 0 & 0 & 0 & 0 \\
\hline Reophax scorpiurus & 4 & 2 & 6 & 0 & 0 & 0 & 0 & 0 & 0 \\
\hline Reophax spp. & 1 & 46 & 47 & 1 & 2 & 3 & 0 & 0 & 0 \\
\hline Rhabdaminna sp. & 2 & 4 & 6 & 0 & 0 & 0 & 0 & 0 & 0 \\
\hline $\begin{array}{l}\text { Spiroplectammina } \\
\text { biformis }\end{array}$ & 6 & 4 & 10 & 0 & 0 & 0 & 0 & 0 & 0 \\
\hline $\begin{array}{l}\text { Spiroplectammina } \\
\text { wrightii }\end{array}$ & 0 & 17 & 17 & 0 & 0 & 0 & 0 & 0 & 0 \\
\hline $\begin{array}{l}\text { Stainforthia } \\
\text { fusiformis }\end{array}$ & 14 & 7 & 21 & 0 & 0 & 0 & 0 & 0 & 0 \\
\hline Textularia earlandi & 2 & 4 & 6 & 0 & 0 & 0 & 0 & 0 & 0 \\
\hline Aggl. varia $^{1}$ & 27 & 11 & 38 & 0 & 0 & 0 & 0 & 0 & 0 \\
\hline Calc. varia $^{2}$ & 40 & 18 & 58 & 6 & 0 & 6 & 0 & 28 & 28 \\
\hline $\begin{array}{l}\text { Total number of } \\
\text { specimens counted }\end{array}$ & 313 & 382 & 695 & 323 & 239 & 562 & 337 & 134 & 471 \\
\hline
\end{tabular}

${ }^{1}$ includes unidentified agglutinated species.

${ }^{2}$ includes unidentified calcareous species.

Table S2) Summary of the percentage of live (stained) and dead foraminifer assemblages at Anholt and Hanö Bay. At C-Ha-1 replicate core tops were collected, noted as $1 \mathrm{a}$ and $1 \mathrm{~b}$. Note that only species with an abundance $>1 \%$ are shown in Fig. 4 .

\begin{tabular}{|l|c|c|c|c|c|c|c|c|c|}
\hline Morphospecies (\%) & $\begin{array}{c}\text { C-An- } \\
\text { 1a Live }\end{array}$ & $\begin{array}{c}\text { C-An-1a } \\
\text { Dead }\end{array}$ & $\begin{array}{c}\text { C-An-1a } \\
\text { Total }\end{array}$ & $\begin{array}{c}\text { C-Ha-1a } \\
\text { Live }\end{array}$ & $\begin{array}{c}\text { C-Ha-1a- } \\
\text { Dead }\end{array}$ & $\begin{array}{c}\text { C-Ha-1a } \\
\text { Total }\end{array}$ & $\begin{array}{c}\text { C-Ha-1b } \\
\text { Live }\end{array}$ & $\begin{array}{c}\text { C-Ha-1b } \\
\text { Dead }\end{array}$ & $\begin{array}{c}\text { C-Ha-1b } \\
\text { Total }\end{array}$ \\
\hline Ammonia sp. (T6) & 0 & 0 & 0 & 13.3 & 0.4 & 7.8 & 11.0 & 0 & 7.9 \\
\hline Ammonia batava & 0 & 0.5 & 0.5 & 0 & 0 & 0 & 0 & 0 & 0 \\
\hline
\end{tabular}




\begin{tabular}{|c|c|c|c|c|c|c|c|c|c|}
\hline Ammotium cassis & 0 & 0.5 & 0 & 0 & 0 & 0 & 0 & 0 & 0 \\
\hline $\begin{array}{l}\text { Ammonia } \\
\text { falsobeccarii }\end{array}$ & 1.0 & 3.1 & 2 & 0 & 0 & 0 & 0 & 0 & 0 \\
\hline $\begin{array}{l}\text { Adercotryma } \\
\text { glomerata }\end{array}$ & 0 & 1.8 & 1 & 0 & 0 & 0 & 0 & 0 & 0 \\
\hline $\begin{array}{l}\text { Ammoscalaria } \\
\text { pseudospiralis }\end{array}$ & 1.3 & 1.0 & 1 & 0 & 0 & 0 & 0 & 0 & 0 \\
\hline Ammodiscus sp. & 0 & 0.3 & 0 & 0 & 0 & 0 & 0 & 0 & 0 \\
\hline $\begin{array}{l}\text { Bulimina } \\
\text { marginata }\end{array}$ & 8.6 & 30.6 & 21 & 0 & 0 & 0 & 0 & 0 & 0 \\
\hline $\begin{array}{l}\text { Bolivina } \\
\text { pseudopunctata }\end{array}$ & 0 & 0 & 0 & 0 & 0 & 0 & 0 & 0 & 0 \\
\hline $\begin{array}{l}\text { Cribrostomoides } \\
\text { jeffreysi }\end{array}$ & 0.3 & 0.8 & 1 & 0 & 0 & 0 & 0 & 0 & 0 \\
\hline $\begin{array}{l}\text { Cassidulina } \\
\text { laevigata }\end{array}$ & 11.2 & 0 & 5 & 0 & 0 & 0 & 0 & 0 & 0 \\
\hline $\begin{array}{l}\text { Cibicidoides } \\
\text { lobatulus }\end{array}$ & 0 & 0.8 & 0 & 0 & 0 & 0 & 0 & 0 & 0 \\
\hline $\begin{array}{l}\text { Cibicides/Cibicidoid } \\
\text { es spp. }\end{array}$ & 0 & 0.3 & 0 & 0 & 0 & 0 & 0 & 0 & 0 \\
\hline $\begin{array}{l}\text { E. clavatum- } \\
\text { selseyense complex }\end{array}$ & 0.3 & 12.6 & 7 & 84.5 & 98.7 & 90.6 & 89.0 & 79.1 & 86.2 \\
\hline $\begin{array}{l}\text { Eggerelloides } \\
\text { medius/scaber }\end{array}$ & 3.8 & 10.7 & 8 & 0 & 0 & 0 & 0 & 0 & 0 \\
\hline Lagena sp. & 0 & 0.3 & 0 & 0 & 0 & 0 & 0 & 0 & 0 \\
\hline $\begin{array}{l}\text { Lagenammina } \\
\text { difflugiformis }\end{array}$ & 0 & 2.4 & 1 & 0 & 0 & 0 & 0 & 0 & 0 \\
\hline Leptohalysis scotti & 2.9 & 0.3 & 1 & 0 & 0 & 0 & 0 & 0 & 0 \\
\hline $\begin{array}{l}\text { Miliolinella } \\
\text { subrotunda }\end{array}$ & 2.6 & 0.3 & 1 & 0 & 0 & 0 & 0 & 0 & 0 \\
\hline $\begin{array}{l}\text { Nonionella aff. } \\
\text { stella }\end{array}$ & 14.1 & 0 & 6 & 0 & 0 & 0 & 0 & 0 & 0 \\
\hline $\begin{array}{l}\text { Nonionellina } \\
\text { labradorica }\end{array}$ & 4.8 & 4.2 & 4 & 0 & 0 & 0 & 0 & 0 & 0 \\
\hline Nonionella turgida & 17.9 & 0 & 8 & 0 & 0 & 0 & 0 & 0 & 0 \\
\hline Pyrgo williamsoni & 0.6 & 0 & 0 & 0 & 0 & 0 & 0 & 0 & 0 \\
\hline
\end{tabular}




\begin{tabular}{|l|c|c|c|c|c|c|c|c|c|}
\hline Reophax scorpiurus & 1.3 & 0.5 & 1 & 0 & 0 & 0 & 0 & 0 & 0 \\
\hline Reophax spp. & 0.3 & 12.0 & 7 & 0.3 & 0.8 & 0.5 & 0 & 0 & 0 \\
\hline Rhabdaminna sp. & 0.6 & 1.0 & 1 & 0 & 0 & 0 & 0 & 0 & 0 \\
\hline $\begin{array}{l}\text { Spiroplectammina } \\
\text { biformis }\end{array}$ & 1.9 & 1.0 & 1 & 0 & 0 & 0 & 0 & 0 & 0 \\
\hline $\begin{array}{l}\text { Spiroplectammina } \\
\text { wrightii }\end{array}$ & 0 & 4.5 & 2 & 0 & 0 & 0 & 0 & 0 & 0 \\
\hline $\begin{array}{l}\text { Stainforthia } \\
\text { fusiformis }\end{array}$ & 4.5 & 1.8 & 3 & 0 & 0 & 0 & 0 & 0 & 0 \\
\hline Textularia earlandi & 0.6 & 1.0 & 1 & 0 & 0 & 0 & 0 & 0 & 0 \\
\hline Aggl. varia & 8.6 & 2.9 & 5 & 0 & 0 & 0 & 0 & 0 & 0 \\
\hline Calc. varia & 12.8 & 4.7 & 8 & 1.9 & 0 & 1.1 & 0 & 20.9 & 5.9 \\
\hline $\begin{array}{l}\text { Total number of } \\
\text { specimens counted }\end{array}$ & 313 & 382 & 695 & 323 & 239 & 562 & 337 & 134 & 471 \\
\hline
\end{tabular}

${ }^{1}$ includes unidentified agglutinated species.

${ }^{2}$ includes unidentified calcareous species.

Table S3) Site location and environmental data for sequenced/imaged specimens.

\begin{tabular}{|c|c|c|c|c|c|c|c|c|c|c|}
\hline $\begin{array}{c}\text { DNA } \\
\text { isolate }\end{array}$ & Genetic type & Station\# & Location & $\begin{array}{c}\text { Date } \\
\text { collection }\end{array}$ & Latitude & Longitude & $\begin{array}{c}\text { Depth } \\
(\mathbf{m})\end{array}$ & $\begin{array}{c}\text { Temperature } \\
\left({ }^{\circ} \mathbf{C}\right)\end{array}$ & $\begin{array}{c}\text { Salinity } \\
\text { Sampling } \\
\text { method }\end{array}$ \\
\hline BA2 & Elphidium S4 & C-Ha-1 & Hanö Bay & $06-09-12$ & $55^{\circ} 38.0^{\prime} \mathrm{N}$ & $14^{\circ} 50.0^{\prime} \mathrm{E}$ & 71 & 5.03 & 13.79 & box core \\
\hline BA4 & Elphidium S4 & C-Ha-1 & Hanö Bay & $06-09-12$ & $55^{\circ} 38.0^{\prime} \mathrm{N}$ & $14^{\circ} 50.0^{\prime} \mathrm{E}$ & 71 & 5.03 & 13.79 & box core \\
\hline BA6 & Elphidium S4 & C-Ha-1 & Hanö Bay & $06-09-12$ & $55^{\circ} 38.0^{\prime} \mathrm{N}$ & $14^{\circ} 50.0^{\prime} \mathrm{E}$ & 71 & 5.03 & 13.79 & box core \\
\hline BA7 & Elphidium S4 & C-Ha-1 & Hanö Bay & $06-09-12$ & $55^{\circ} 38.0^{\prime} \mathrm{N}$ & $14^{\circ} 50.0^{\prime} \mathrm{E}$ & 71 & 5.03 & 13.79 & box core \\
\hline BA9 & Elphidium S4 & C-Ha-1 & Hanö Bay & $06-09-12$ & $55^{\circ} 38.0^{\prime} \mathrm{N}$ & $14^{\circ} 50.0^{\prime} \mathrm{E}$ & 71 & 5.03 & 13.79 & box core \\
\hline BA10 & Elphidium S4 & C-Ha-1 & Hanö Bay & $06-09-12$ & $55^{\circ} 38.0^{\prime} \mathrm{N}$ & $14^{\circ} 50.0^{\prime} \mathrm{E}$ & 71 & 5.03 & 13.79 & box core \\
\hline
\end{tabular}




\begin{tabular}{|c|c|c|c|c|c|c|c|c|c|c|}
\hline BA12 & Ammonia T6 & C-Ha-1 & Hanö Bay & $06-09-12$ & $55^{\circ} 38.0^{\prime} \mathrm{N}$ & $14^{\circ} 50.0^{\prime} \mathrm{E}$ & 71 & 5.03 & 13.79 & box core \\
\hline BA13 & Ammonia T6 & C-Ha-1 & Hanö Bay & 06-09-12 & $55^{\circ} 38.0^{\prime} \mathrm{N}$ & $14^{\circ} 50.0^{\prime} \mathrm{E}$ & 71 & 5.03 & 13.79 & box core \\
\hline BA14 & Ammonia T6 & C-Ha-1 & Hanö Bay & $06-09-12$ & $55^{\circ} 38.0^{\prime} \mathrm{N}$ & $14^{\circ} 50.0^{\prime} \mathrm{E}$ & 71 & 5.03 & 13.79 & box core \\
\hline BA15 & Ammonia T6 & C-Ha-1 & Hanö Bay & 06-09-12 & $55^{\circ} 38.0^{\prime} \mathrm{N}$ & $14^{\circ} 50.0^{\prime} \mathrm{E}$ & 71 & 5.03 & 13.79 & box core \\
\hline BA16 & Ammonia T6 & C-Ha-1 & Hanö Bay & 06-09-12 & $55^{\circ} 38.0^{\prime} \mathrm{N}$ & $14^{\circ} 50.0^{\prime} \mathrm{E}$ & 71 & 5.03 & 13.79 & box core \\
\hline BA17 & Ammonia T6 & C-Ha-1 & Hanö Bay & 06-09-12 & $55^{\circ} 38.0^{\prime} \mathrm{N}$ & $14^{\circ} 50.0^{\prime} \mathrm{E}$ & 71 & 5.03 & 13.79 & box core \\
\hline BA18 & Ammonia T6 & C-Ha-1 & Hanö Bay & 06-09-12 & $55^{\circ} 38.0^{\prime} \mathrm{N}$ & $14^{\circ} 50.0^{\prime} \mathrm{E}$ & 71 & 5.03 & 13.79 & box core \\
\hline BA19 & Ammonia T6 & C-Ha-1 & Hanö Bay & $06-09-12$ & $55^{\circ} 38.0^{\prime} \mathrm{N}$ & $14^{\circ} 50.0^{\prime} \mathrm{E}$ & 71 & 5.03 & 13.79 & box core \\
\hline BA20 & Ammonia T6 & C-Ha-1 & Hanö Bay & 06-09-12 & $55^{\circ} 38.0^{\prime} \mathrm{N}$ & $14^{\circ} 50.0^{\prime} \mathrm{E}$ & 71 & 5.03 & 13.79 & box core \\
\hline BA21 & Cassidulina sp. & C-An-1 & Anholt & 04-09-12 & $56^{\circ} 26.048^{\prime} \mathrm{N}$ & $11^{\circ} 50.043^{\prime} \mathrm{E}$ & 30 & 9.58 & 32.86 & box core \\
\hline BA22 & Cassidulina sp. & C-An-1 & Anholt & 04-09-12 & $56^{\circ} 26.048^{\prime} \mathrm{N}$ & $11^{\circ} 50.043^{\prime} \mathrm{E}$ & 30 & 9.58 & 32.86 & box core \\
\hline BA23 & Cassidulina sp. & C-An-1 & Anholt & 04-09-12 & $56^{\circ} 26.048^{\prime} \mathrm{N}$ & $11^{\circ} 50.043^{\prime} \mathrm{E}$ & 30 & 9.58 & 32.86 & box core \\
\hline BA24 & Cassidulina sp. & C-An-1 & Anholt & 04-09-12 & $56^{\circ} 26.048^{\prime} \mathrm{N}$ & $11^{\circ} 50.043^{\prime} \mathrm{E}$ & 30 & 9.58 & 32.86 & box core \\
\hline BA25 & Cassidulina sp. & C-An-1 & Anholt & 04-09-12 & $56^{\circ} 26.048^{\prime} \mathrm{N}$ & $11^{\circ} 50.043^{\prime} \mathrm{E}$ & 30 & 9.58 & 32.86 & box core \\
\hline BA26 & Cassidulina sp. & C-An-1 & Anholt & 04-09-12 & $56^{\circ} 26.048^{\prime} \mathrm{N}$ & $11^{\circ} 50.043^{\prime} \mathrm{E}$ & 30 & 9.58 & 32.86 & box core \\
\hline BA29 & Bulimina sp. & C-An-1 & Anholt & 04-09-12 & $56^{\circ} 26.048^{\prime} \mathrm{N}$ & $11^{\circ} 50.043^{\prime} \mathrm{E}$ & 30 & 9.58 & 32.86 & box core \\
\hline BA30 & Bulimina sp. & C-An-1 & Anholt & 04-09-12 & $56^{\circ} 26.048^{\prime} \mathrm{N}$ & $11^{\circ} 50.043^{\prime} \mathrm{E}$ & 30 & 9.58 & 32.86 & box core \\
\hline BA34 & Bulimina sp. & C-An-1 & Anholt & 04-09-12 & $56^{\circ} 26.048^{\prime} \mathrm{N}$ & $11^{\circ} 50.043^{\prime} \mathrm{E}$ & 30 & 9.58 & 32.86 & box core \\
\hline BA36 & Cassidulina sp. & C-An-1 & Anholt & 04-09-12 & $56^{\circ} 26.048^{\prime} \mathrm{N}$ & $11^{\circ} 50.043^{\prime} \mathrm{E}$ & 30 & 9.58 & 32.86 & box core \\
\hline BA37 & N. labradorica & C-An-1 & Anholt & 04-09-12 & $56^{\circ} 26.048^{\prime} \mathrm{N}$ & $11^{\circ} 50.043^{\prime} \mathrm{E}$ & 30 & 9.58 & 32.86 & box core \\
\hline BA38 & Ammonia T3 & C-An-1 & Anholt & 04-09-12 & $56^{\circ} 26.048^{\prime} \mathrm{N}$ & $11^{\circ} 50.043^{\prime} \mathrm{E}$ & 30 & 9.58 & 32.86 & box core \\
\hline BA45 & Bulimina sp. & C-An-1 & Anholt & 04-09-12 & $56^{\circ} 26.048^{\prime} \mathrm{N}$ & $11^{\circ} 50.043^{\prime} \mathrm{E}$ & 30 & 9.58 & 32.86 & box core \\
\hline
\end{tabular}




\begin{tabular}{|c|c|c|c|c|c|c|c|c|c|c|}
\hline BA48 & Bulimina sp. & C-An-1 & Anholt & 04-09-12 & $56^{\circ} 26.048^{\prime} \mathrm{N}$ & $11^{\circ} 50.043^{\prime} \mathrm{E}$ & 30 & 9.58 & 32.86 & box core \\
\hline BA51 & Cassidulina sp. & C-An-1 & Anholt & 04-09-12 & $56^{\circ} 26.048^{\prime} \mathrm{N}$ & $11^{\circ} 50.043^{\prime} \mathrm{E}$ & 30 & 9.58 & 32.86 & box core \\
\hline BA53 & Cassidulina sp. & $\begin{array}{l}\text { C-An-1 } \\
\text { (n) }\end{array}$ & Anholt & 04-09-12 & $56^{\circ} 26.048^{\prime} \mathrm{N}$ & $11^{\circ} 50.043^{\prime} \mathrm{E}$ & 30 & 9.58 & 32.86 & box core \\
\hline BA54 & Cassidulina sp. & C-An-1 & Anholt & 04-09-12 & $56^{\circ} 26.048^{\prime} \mathrm{N}$ & $11^{\circ} 50.043^{\prime} \mathrm{E}$ & 30 & 9.58 & 32.86 & box core \\
\hline BA55 & Cassidulina sp. & C-An-1 & Anholt & 04-09-12 & $56^{\circ} 26.048^{\prime} \mathrm{N}$ & $11^{\circ} 50.043^{\prime} \mathrm{E}$ & 30 & 9.58 & 32.86 & box core \\
\hline BA56 & Cassidulina sp. & C-An-1 & Anholt & 04-09-12 & $56^{\circ} 26.048^{\prime} \mathrm{N}$ & $11^{\circ} 50.043^{\prime} \mathrm{E}$ & 30 & 9.58 & 32.86 & box core \\
\hline BA58 & Cassidulina sp. & C-An-1 & Anholt & 04-09-12 & $56^{\circ} 26.048^{\prime} \mathrm{N}$ & $11^{\circ} 50.043^{\prime} \mathrm{E}$ & 30 & 9.58 & 32.86 & box core \\
\hline BA65 & Elphidium S4 & C-An-1 & Anholt & 04-09-12 & $56^{\circ} 26.048^{\prime} \mathrm{N}$ & $11^{\circ} 50.043^{\prime} \mathrm{E}$ & 30 & 9.58 & 32.86 & box core \\
\hline BA66 & Elphidium S4 & C-An-1 & Anholt & 04-09-12 & $56^{\circ} 26.048^{\prime} \mathrm{N}$ & $11^{\circ} 50.043^{\prime} \mathrm{E}$ & 30 & 9.58 & 32.86 & box core \\
\hline BA67 & Elphidium S4 & C-Ha-1 & Hanö Bay & $\begin{array}{l}06-09-12 \\
\end{array}$ & $55^{\circ} 38.0^{\prime} \mathrm{N}$ & $14^{\circ} 50.0^{\prime} \mathrm{E}$ & 71 & 5.03 & 13.79 & box core \\
\hline BA68 & Elphidium S4 & C-Ha-1 & Hanö Bay & 06-09-12 & $55^{\circ} 38.0^{\prime} \mathrm{N}$ & $14^{\circ} 50.0^{\prime} \mathrm{E}$ & 71 & 5.03 & 13.79 & box core \\
\hline BA69 & Elphidium S4 & C-Ha-1 & Hanö Bay & 06-09-12 & $55^{\circ} 38.0^{\prime} \mathrm{N}$ & $14^{\circ} 50.0^{\prime} \mathrm{E}$ & 71 & 5.03 & 13.79 & box core \\
\hline BA70 & Elphidium S4 & C-Ha-1 & Hanö Bay & 06-09-12 & $55^{\circ} 38.0^{\prime} \mathrm{N}$ & $14^{\circ} 50.0^{\prime} \mathrm{E}$ & 71 & 5.03 & 13.79 & box core \\
\hline BA71 & Elphidium S4 & C-Ha-1 & Hanö Bay & 06-09-12 & $55^{\circ} 38.0^{\prime} \mathrm{N}$ & $14^{\circ} 50.0^{\prime} \mathrm{E}$ & 71 & 5.03 & 13.79 & box core \\
\hline BA72 & Elphidium S4 & C-Ha-1 & Hanö Bay & 06-09-12 & $55^{\circ} 38.0^{\prime} \mathrm{N}$ & $14^{\circ} 50.0^{\prime} \mathrm{E}$ & 71 & 5.03 & 13.79 & box core \\
\hline BA73 & Elphidium S4 & C-Ha-1 & Hanö Bay & 06-09-12 & $55^{\circ} 38.0^{\prime} \mathrm{N}$ & $14^{\circ} 50.0^{\prime} \mathrm{E}$ & 71 & 5.03 & 13.79 & box core \\
\hline BA75 & Ammonia T6 & C-Ha-1 & Hanö Bay & 06-09-12 & $55^{\circ} 38.0^{\prime} \mathrm{N}$ & $14^{\circ} 50.0^{\prime} \mathrm{E}$ & 71 & 5.03 & 13.79 & box core \\
\hline BA76 & Ammonia T6 & C-Ha-1 & Hanö Bay & $06-09-12$ & $55^{\circ} 38.0^{\prime} \mathrm{N}$ & $14^{\circ} 50.0^{\prime} \mathrm{E}$ & 71 & 5.03 & 13.79 & box core \\
\hline BA77 & Ammonia T6 & C-Ha-1 & Hanö Bay & 06-09-12 & $55^{\circ} 38.0^{\prime} \mathrm{N}$ & $14^{\circ} 50.0^{\prime} \mathrm{E}$ & 71 & 5.03 & 13.79 & box core \\
\hline BA78 & Ammonia T6 & C-Ha-1 & Hanö Bay & $\begin{array}{c}06-09-12 \\
\end{array}$ & $55^{\circ} 38.0^{\prime} \mathrm{N}$ & $14^{\circ} 50.0^{\prime} \mathrm{E}$ & 71 & 5.03 & 13.79 & box core \\
\hline BA80 & Ammonia T6 & C-Ha-1 & Hanö Bay & $06-09-12$ & $55^{\circ} 38.0^{\prime} \mathrm{N}$ & $14^{\circ} 50.0^{\prime} \mathrm{E}$ & 71 & 5.03 & 13.79 & box core \\
\hline BA81 & Elphidium S4 & C-Ha-2 & Hanö Bay & 06-09-12 & $55^{\circ} 57.976^{\prime} \mathrm{N}$ & $14^{\circ} .49 .659^{\prime} \mathrm{E}$ & 50 & 5.28 & 10.44 & box core \\
\hline
\end{tabular}




\begin{tabular}{|c|c|c|c|c|c|c|c|c|c|c|}
\hline BA82 & Elphidium S4 & C-Ha-2 & Hanö Bay & 06-09-12 & $55^{\circ} 57.976^{\prime} \mathrm{N}$ & $14^{\circ} .49 .659^{\prime} \mathrm{E}$ & 50 & 5.28 & 10.44 & box core \\
\hline BA83 & Elphidium S4 & C-Ha-2 & Hanö Bay & 06-09-12 & $55^{\circ} 57.976^{\prime} \mathrm{N}$ & $14^{\circ} .49 .659^{\prime} \mathrm{E}$ & 50 & 5.28 & 10.44 & box core \\
\hline BA84 & Elphidium S4 & C-Ha-2 & Hanö Bay & 06-09-12 & $55^{\circ} 57.976^{\prime} \mathrm{N}$ & $14^{\circ} .49 .659^{\prime} \mathrm{E}$ & 50 & 5.28 & 10.44 & box core \\
\hline BA85 & Elphidium S4 & C-Ha-2 & Hanö Bay & 06-09-12 & $55^{\circ} 57.976^{\prime} \mathrm{N}$ & $14^{\circ} .49 .659^{\prime} \mathrm{E}$ & 50 & 5.28 & 10.44 & box core \\
\hline BA86 & Elphidium S4 & C-Ha-2 & Hanö Bay & 06-09-12 & $55^{\circ} 57.976^{\prime} \mathrm{N}$ & $14^{\circ} .49 .659^{\prime} \mathrm{E}$ & 50 & 5.28 & 10.44 & box core \\
\hline BA87 & Elphidium S4 & C-Ha-2 & Hanö Bay & 06-09-12 & $55^{\circ} 57.976^{\prime} \mathrm{N}$ & $14^{\circ} .49 .659^{\prime} \mathrm{E}$ & 50 & 5.28 & 10.44 & box core \\
\hline BA88 & Elphidium S4 & C-Ha-2 & Hanö Bay & 06-09-12 & $55^{\circ} 57.976^{\prime} \mathrm{N}$ & $14^{\circ} .49 .659^{\prime} \mathrm{E}$ & 50 & 5.28 & 10.44 & box core \\
\hline BA89 & Elphidium S4 & C-Ha-2 & Hanö Bay & 06-09-12 & $55^{\circ} 57.976^{\prime} \mathrm{N}$ & $14^{\circ} .49 .659^{\prime} \mathrm{E}$ & 50 & 5.28 & 10.44 & box core \\
\hline BA90 & Elphidium S4 & C-Ha-2 & Hanö Bay & 06-09-12 & $55^{\circ} 57.976^{\prime} \mathrm{N}$ & $14^{\circ} .49 .659^{\prime} \mathrm{E}$ & 50 & 5.28 & 10.44 & box core \\
\hline BA91 & Elphidium S4 & C-Ha-2 & Hanö Bay & 06-09-12 & $55^{\circ} 57.976^{\prime} \mathrm{N}$ & $14^{\circ} .49 .659^{\prime} \mathrm{E}$ & 50 & 5.28 & 10.44 & box core \\
\hline BA92 & Elphidium S4 & C-Ha-2 & Hanö Bay & 06-09-12 & $55^{\circ} 57.976^{\prime} \mathrm{N}$ & $14^{\circ} .49 .659^{\prime} \mathrm{E}$ & 50 & 5.28 & 10.44 & box core \\
\hline BA93 & Elphidium S4 & C-Ha-2 & Hanö Bay & 06-09-12 & $55^{\circ} 57.976^{\prime} \mathrm{N}$ & $14^{\circ} .49 .659^{\prime} \mathrm{E}$ & 50 & 5.28 & 10.44 & box core \\
\hline BA94 & Elphidium S4 & C-Ha-2 & Hanö Bay & 06-09-12 & $55^{\circ} 57.976^{\prime} \mathrm{N}$ & $14^{\circ} .49 .659^{\prime} \mathrm{E}$ & 50 & 5.28 & 10.44 & box core \\
\hline BA95 & Elphidium S4 & C-Ha-2 & Hanö Bay & 06-09-12 & $55^{\circ} 57.976^{\prime} \mathrm{N}$ & $14^{\circ} .49 .659^{\prime} \mathrm{E}$ & 50 & 5.28 & 10.44 & box core \\
\hline BA96 & Elphidium S4 & C-Ha-2 & Hanö Bay & 06-09-12 & $55^{\circ} 57.976^{\prime} \mathrm{N}$ & $14^{\circ} .49 .659^{\prime} \mathrm{E}$ & 50 & 5.28 & 10.44 & box core \\
\hline BA97 & Elphidium S4 & C-Ha-2 & Hanö Bay & 06-09-12 & $55^{\circ} 57.976^{\prime} \mathrm{N}$ & $14^{\circ} .49 .659^{\prime} \mathrm{E}$ & 50 & 5.28 & 10.44 & box core \\
\hline BA98 & Elphidium S4 & C-Ha-2 & Hanö Bay & 06-09-12 & $55^{\circ} 57.976^{\prime} \mathrm{N}$ & $14^{\circ} .49 .659^{\prime} \mathrm{E}$ & 50 & 5.28 & 10.44 & box core \\
\hline BA99 & Elphidium S4 & C-Ha-2 & Hanö Bay & 06-09-12 & $55^{\circ} 57.976^{\prime} \mathrm{N}$ & $14^{\circ} .49 .659^{\prime} \mathrm{E}$ & 50 & 5.28 & 10.44 & box core \\
\hline BA100 & Elphidium S4 & C-Ha-2 & Hanö Bay & 06-09-12 & $55^{\circ} 57.976^{\prime} \mathrm{N}$ & $14^{\circ} .49 .659^{\prime} \mathrm{E}$ & 50 & 5.28 & 10.44 & box core \\
\hline BA102 & Elphidium S4 & C-Ha-2 & Hanö Bay & 06-09-12 & $55^{\circ} 57.976^{\prime} \mathrm{N}$ & $14^{\circ} .49 .659^{\prime} \mathrm{E}$ & 50 & 5.28 & 10.44 & box core \\
\hline BA103 & Elphidium S4 & C-Ha-2 & Hanö Bay & 06-09-12 & $55^{\circ} 57.976^{\prime} \mathrm{N}$ & $14^{\circ} .49 .659^{\prime} \mathrm{E}$ & 50 & 5.28 & 10.44 & box core \\
\hline BA105 & Elphidium S4 & C-Ha-2 & Hanö Bay & 06-09-12 & $55^{\circ} 57.976^{\prime} \mathrm{N}$ & $14^{\circ} .49 .659^{\prime} \mathrm{E}$ & 50 & 5.28 & 10.44 & box core \\
\hline
\end{tabular}




\begin{tabular}{|c|c|c|c|c|c|c|c|c|c|c|}
\hline $\begin{array}{l}\text { BA106 } \\
\end{array}$ & Elphidium S4 & C-Ha-2 & Hanö Bay & 06-09-12 & $55^{\circ} 57.976^{\prime} \mathrm{N}$ & $14^{\circ} .49 .659^{\prime} \mathrm{E}$ & 50 & 5.28 & 10.44 & box core \\
\hline BA107 & Elphidium S4 & C-Ha-2 & Hanö Bay & 06-09-12 & $55^{\circ} 57.976^{\prime} \mathrm{N}$ & $14^{\circ} .49 .659^{\prime} \mathrm{E}$ & 50 & 5.28 & 10.44 & box core \\
\hline $\begin{array}{l}\text { BA108 } \\
\end{array}$ & Elphidium S7 & C-Ha-2 & Hanö Bay & 06-09-12 & $55^{\circ} 57.976^{\prime} \mathrm{N}$ & $14^{\circ} .49 .659^{\prime} \mathrm{E}$ & 50 & 5.28 & 10.44 & box core \\
\hline $\begin{array}{l}\text { BA109 } \\
\end{array}$ & Elphidium S4 & C-Ha-2 & Hanö Bay & $\begin{array}{l}06-09-12 \\
\end{array}$ & $55^{\circ} 57.976 ' \mathrm{~N}$ & $14^{\circ} .49 .659^{\prime} \mathrm{E}$ & 50 & 5.28 & 10.44 & box core \\
\hline $\begin{array}{l}\text { BA110 } \\
\end{array}$ & Elphidium S4 & C-Ha-2 & Hanö Bay & 06-09-12 & $55^{\circ} 57.976 ' \mathrm{~N}$ & $14^{\circ} .49 .659^{\prime} \mathrm{E}$ & 50 & 5.28 & 10.44 & box core \\
\hline BA111 & Elphidium S4 & C-Ha-2 & Hanö Bay & 06-09-12 & $55^{\circ} 57.976^{\prime} \mathrm{N}$ & $14^{\circ} .49 .659^{\prime} \mathrm{E}$ & 50 & 5.28 & 10.44 & box core \\
\hline BA112 & Elphidium S4 & C-Ha-2 & Hanö Bay & 06-09-12 & $55^{\circ} 57.976^{\prime} \mathrm{N}$ & $14^{\circ} .49 .659^{\prime} \mathrm{E}$ & 50 & 5.28 & 10.44 & box core \\
\hline BA113 & Elphidium S4 & C-Ha-2 & Hanö Bay & $\begin{array}{l}06-09-12 \\
\end{array}$ & $55^{\circ} 57.976^{\prime} \mathrm{N}$ & $14^{\circ} .49 .659^{\prime} \mathrm{E}$ & 50 & 5.28 & 10.44 & box core \\
\hline BA114 & Elphidium S4 & C-Ha-2 & Hanö Bay & 06-09-12 & $55^{\circ} 57.976^{\prime} \mathrm{N}$ & $14^{\circ} .49 .659^{\prime} \mathrm{E}$ & 50 & 5.28 & 10.44 & box core \\
\hline BA115 & Elphidium S4 & C-Ha-2 & Hanö Bay & 06-09-12 & $55^{\circ} 57.976^{\prime} \mathrm{N}$ & $14^{\circ} .49 .659^{\prime} \mathrm{E}$ & 50 & 5.28 & 10.44 & box core \\
\hline BA116 & Elphidium S4 & C-Ha-2 & Hanö Bay & $\begin{array}{l}06-09-12 \\
\end{array}$ & $55^{\circ} 57.976^{\prime} \mathrm{N}$ & $14^{\circ} .49 .659^{\prime} \mathrm{E}$ & 50 & 5.28 & 10.44 & box core \\
\hline BA117 & Elphidium S4 & C-Ha-2 & Hanö Bay & 06-09-12 & $55^{\circ} 57.976^{\prime} \mathrm{N}$ & $14^{\circ} .49 .659^{\prime} \mathrm{E}$ & 50 & 5.28 & 10.44 & box core \\
\hline BA118 & Elphidium S4 & C-Ha-2 & Hanö Bay & $\begin{array}{l}06-09-12 \\
\end{array}$ & $55^{\circ} 57.976^{\prime} \mathrm{N}$ & $14^{\circ} .49 .659^{\prime} \mathrm{E}$ & 50 & 5.28 & 10.44 & box core \\
\hline BA119 & Elphidium S4 & С-Ha-2 & Hanö Bay & 06-09-12 & $55^{\circ} 57.976^{\prime} \mathrm{N}$ & $14^{\circ} .49 .659^{\prime} \mathrm{E}$ & 50 & 5.28 & 10.44 & box core \\
\hline BA121 & Elphidium S4 & C-Ha-2 & Hanö Bay & $\begin{array}{l}06-09-12 \\
\end{array}$ & $55^{\circ} 57.976^{\prime} \mathrm{N}$ & $14^{\circ} .49 .659^{\prime} \mathrm{E}$ & 50 & 5.28 & 10.44 & box core \\
\hline BA122 & Elphidium S4 & C-Ha-2 & Hanö Bay & 06-09-12 & $55^{\circ} 57.976^{\prime} \mathrm{N}$ & $14^{\circ} .49 .659^{\prime} \mathrm{E}$ & 50 & 5.28 & 10.44 & box core \\
\hline BA123 & Elphidium S4 & C-Ha-2 & Hanö Bay & 06-09-12 & $55^{\circ} 57.976^{\prime} \mathrm{N}$ & $14^{\circ} .49 .659^{\prime} \mathrm{E}$ & 50 & 5.28 & 10.44 & box core \\
\hline BA124 & Elphidium S4 & C-Ha-2 & Hanö Bay & 06-09-12 & $55^{\circ} 57.976^{\prime} \mathrm{N}$ & $14^{\circ} .49 .659^{\prime} \mathrm{E}$ & 50 & 5.28 & 10.44 & box core \\
\hline BA125 & Elphidium S4 & C-Ha-2 & Hanö Bay & 06-09-12 & $55^{\circ} 57.976^{\prime} \mathrm{N}$ & $14^{\circ} .49 .659^{\prime} \mathrm{E}$ & 50 & 5.28 & 10.44 & box core \\
\hline BA126 & Elphidium S4 & C-Ha-2 & Hanö Bay & 06-09-12 & $55^{\circ} 57.976^{\prime} \mathrm{N}$ & $14^{\circ} .49 .659^{\prime} \mathrm{E}$ & 50 & 5.28 & 10.44 & box core \\
\hline BA127 & Elphidium S7 & C-Ha-2 & Hanö Bay & 06-09-12 & $55^{\circ} 57.976^{\prime} \mathrm{N}$ & $14^{\circ} .49 .659^{\prime} \mathrm{E}$ & 50 & 5.28 & 10.44 & box core \\
\hline BA128 & Elphidium S4 & C-Ha-2 & Hanö Bay & 06-09-12 & $55^{\circ} 57.976^{\prime} \mathrm{N}$ & $14^{\circ} .49 .659^{\prime} \mathrm{E}$ & 50 & 5.28 & 10.44 & box core \\
\hline
\end{tabular}




\begin{tabular}{|c|c|c|c|c|c|c|c|c|c|c|}
\hline BA129 & Elphidium S4 & C-Ha-2 & Hanö Bay & 06-09-12 & $55^{\circ} 57.976^{\prime} \mathrm{N}$ & $14^{\circ} .49 .659^{\prime} \mathrm{E}$ & 50 & 5.28 & 10.44 & box core \\
\hline BA130 & Elphidium S4 & C-Ha-2 & Hanö Bay & 06-09-12 & $55^{\circ} 57.976^{\prime} \mathrm{N}$ & $14^{\circ} .49 .659^{\prime} \mathrm{E}$ & 50 & 5.28 & 10.44 & box core \\
\hline BA131 & Elphidium S4 & C-Ha-2 & Hanö Bay & 06-09-12 & $55^{\circ} 57.976^{\prime} \mathrm{N}$ & $14^{\circ} .49 .659^{\prime} \mathrm{E}$ & 50 & 5.28 & 10.44 & box core \\
\hline BA132 & Elphidium S4 & C-Ha-2 & Hanö Bay & $\begin{array}{l}06-09-12 \\
\end{array}$ & $55^{\circ} 57.976 \mathrm{~N}$ & $14^{\circ} .49 .659^{\prime} \mathrm{E}$ & 50 & 5.28 & 10.44 & box core \\
\hline BA133 & Elphidium S4 & C-Ha-2 & Hanö Bay & 06-09-12 & $55^{\circ} 57.976 \mathrm{~N}$ & $14^{\circ} .49 .659^{\prime} \mathrm{E}$ & 50 & 5.28 & 10.44 & box core \\
\hline BA134 & Elphidium S4 & C-Ha-2 & Hanö Bay & 06-09-12 & $55^{\circ} 57.976^{\prime} \mathrm{N}$ & $14^{\circ} .49 .659^{\prime} \mathrm{E}$ & 50 & 5.28 & 10.44 & box core \\
\hline BA135 & Elphidium S4 & C-Ha-2 & Hanö Bay & 06-09-12 & $55^{\circ} 57.976^{\prime} \mathrm{N}$ & $14^{\circ} .49 .659^{\prime} \mathrm{E}$ & 50 & 5.28 & 10.44 & box core \\
\hline BA136 & Elphidium S4 & C-Ha-2 & Hanö Bay & 06-09-12 & $55^{\circ} 57.976 \mathrm{~N}$ & $14^{\circ} .49 .659^{\prime} \mathrm{E}$ & 50 & 5.28 & 10.44 & box core \\
\hline BA137 & Elphidium S4 & C-Ha-2 & Hanö Bay & 06-09-12 & $55^{\circ} 57.976^{\prime} \mathrm{N}$ & $14^{\circ} .49 .659^{\prime} \mathrm{E}$ & 50 & 5.28 & 10.44 & box core \\
\hline BA138 & Elphidium S4 & C-Ha-2 & Hanö Bay & $\begin{array}{l}06-09-12 \\
\end{array}$ & $55^{\circ} 57.976^{\prime} \mathrm{N}$ & $14^{\circ} .49 .659^{\prime} \mathrm{E}$ & 50 & 5.28 & 10.44 & box core \\
\hline BA139 & Elphidium S4 & C-Ha-2 & Hanö Bay & $\begin{array}{l}06-09-12 \\
\end{array}$ & $55^{\circ} 57.976^{\prime} \mathrm{N}$ & $14^{\circ} .49 .659^{\prime} \mathrm{E}$ & 50 & 5.28 & 10.44 & box core \\
\hline BA140 & Elphidium S4 & C-Ha-2 & Hanö Bay & 06-09-12 & $55^{\circ} 57.976^{\prime} \mathrm{N}$ & $14^{\circ} .49 .659^{\prime} \mathrm{E}$ & 50 & 5.28 & 10.44 & box core \\
\hline BA141 & Elphidium S4 & C-Ha-2 & Hanö Bay & $\begin{array}{l}06-09-12 \\
\end{array}$ & $55^{\circ} 57.976^{\prime} \mathrm{N}$ & $14^{\circ} .49 .659^{\prime} \mathrm{E}$ & 50 & 5.28 & 10.44 & box core \\
\hline BA142 & Elphidium S4 & C-Ha-2 & Hanö Bay & 06-09-12 & $55^{\circ} 57.976^{\prime} \mathrm{N}$ & $14^{\circ} .49 .659^{\prime} \mathrm{E}$ & 50 & 5.28 & 10.44 & box core \\
\hline $\begin{array}{l}\text { BA143 } \\
\end{array}$ & Elphidium S7 & C-Ha-2 & Hanö Bay & 06-09-12 & $55^{\circ} 57.976^{\prime} \mathrm{N}$ & $14^{\circ} .49 .659^{\prime} \mathrm{E}$ & 50 & 5.28 & 10.44 & box core \\
\hline BA144 & Elphidium S7 & C-Ha-2 & Hanö Bay & $\begin{array}{l}06-09-12 \\
\end{array}$ & $55^{\circ} 57.976^{\prime} \mathrm{N}$ & $14^{\circ} .49 .659^{\prime} \mathrm{E}$ & 50 & 5.28 & 10.44 & box core \\
\hline BA145 & Ammonia T6 & C-Ha-2 & Hanö Bay & 06-09-12 & $55^{\circ} 57.976^{\prime} \mathrm{N}$ & $14^{\circ} .49 .659^{\prime} \mathrm{E}$ & 50 & 5.28 & 10.44 & box core \\
\hline BA146 & Ammonia T6 & C-Ha-2 & Hanö Bay & $\begin{array}{l}06-09-12 \\
\end{array}$ & $55^{\circ} 57.976^{\prime} \mathrm{N}$ & $14^{\circ} .49 .659^{\prime} \mathrm{E}$ & 50 & 5.28 & 10.44 & box core \\
\hline BA147 & Ammonia T6 & C-Ha-2 & Hanö Bay & 06-09-12 & $55^{\circ} 57.976^{\prime} \mathrm{N}$ & $14^{\circ} .49 .659^{\prime} \mathrm{E}$ & 50 & 5.28 & 10.44 & box core \\
\hline BA148 & Ammonia T6 & C-Ha-2 & Hanö Bay & $\begin{array}{l}06-09-12 \\
\end{array}$ & $55^{\circ} 57.976^{\prime} \mathrm{N}$ & $14^{\circ} .49 .659^{\prime} \mathrm{E}$ & 50 & 5.28 & 10.44 & box core \\
\hline BA149 & Elphidium S4 & DBY2-1 & $\begin{array}{l}\text { Arkona } \\
\text { Basin }\end{array}$ & 11-11-13 & $55^{\circ} 00.000^{\prime} \mathrm{N}$ & $14^{\circ} 04.950^{\prime} \mathrm{E}$ & 46 & 11.73 & 21.6 & box core \\
\hline BA153 & Elphidium S7 & DBY2-1 & Arkona & $11-11-13$ & $55^{\circ} 00.000^{\prime} \mathrm{N}$ & $14^{\circ} 04.950^{\prime} \mathrm{E}$ & 46 & 11.73 & 21.6 & box core \\
\hline
\end{tabular}




\begin{tabular}{|c|c|c|c|c|c|c|c|c|c|c|}
\hline & & & Basin & & & & & & & \\
\hline BA158 & Elphidium S4 & DBY2-1 & $\begin{array}{c}\text { Arkona } \\
\text { Basin }\end{array}$ & 11-11-13 & $55^{\circ} 00.000^{\prime} \mathrm{N}$ & $14^{\circ} 04.950^{\prime} \mathrm{E}$ & 46 & 11.73 & 21.6 & box core \\
\hline BA176 & Elphidium S7 & DBY2-1 & $\begin{array}{c}\text { Arkona } \\
\text { Basin }\end{array}$ & $11-11-13$ & $55^{\circ} 00.000^{\prime} \mathrm{N}$ & $14^{\circ} 04.950^{\prime} \mathrm{E}$ & 46 & 11.73 & 21.6 & box core \\
\hline BA197 & Elphidium S7 & DBY2-1 & $\begin{array}{c}\text { Arkona } \\
\text { Basin }\end{array}$ & 11-11-13 & $55^{\circ} 00.000^{\prime} \mathrm{N}$ & $14^{\circ} 04.950^{\prime} \mathrm{E}$ & 46 & 11.73 & 21.6 & box core \\
\hline BA198 & Elphidium S7 & DBY2-1 & $\begin{array}{c}\text { Arkona } \\
\text { Basin }\end{array}$ & 11-11-13 & $55^{\circ} 00.000^{\prime} \mathrm{N}$ & $14^{\circ} 04.950^{\prime} \mathrm{E}$ & 46 & 11.73 & 21.6 & box core \\
\hline BA207 & Elphidium S4 & DBY2-1 & $\begin{array}{c}\text { Arkona } \\
\text { Basin }\end{array}$ & 11-11-13 & $55^{\circ} 00.000^{\prime} \mathrm{N}$ & $14^{\circ} 04.950^{\prime} \mathrm{E}$ & 46 & 11.73 & 21.6 & box core \\
\hline BA219 & Bulimina sp. & DAn-1A & Anholt & 07-11-13 & $56^{\circ} 40.130^{\prime} \mathrm{N}$ & $12^{\circ} 07.000^{\prime} \mathrm{E}$ & 61 & 9.21 & 34.49 & box core \\
\hline $\begin{array}{l}\text { BA220 } \\
\end{array}$ & Bulimina sp. & DAn-1A & Anholt & 07-11-13 & $56^{\circ} 40.130^{\prime} \mathrm{N}$ & $12^{\circ} 07.000^{\prime} \mathrm{E}$ & 61 & 9.21 & 34.49 & box core \\
\hline BA221 & Bulimina sp. & DAn-1A & Anholt & 07-11-13 & $56^{\circ} 40.130^{\prime} \mathrm{N}$ & $12^{\circ} 07.000^{\prime} \mathrm{E}$ & 61 & 9.21 & 34.49 & box core \\
\hline$\overline{\text { BA222 }}$ & Bulimina sp. & DAn-1A & Anholt & 07-11-13 & $56^{\circ} 40.130^{\prime} \mathrm{N}$ & $12^{\circ} 07.000^{\prime} \mathrm{E}$ & 61 & 9.21 & 34.49 & box core \\
\hline BA225 & Bulimina sp. & DAn-1A & Anholt & 07-11-13 & $56^{\circ} 40.130^{\prime} \mathrm{N}$ & $12^{\circ} 07.000^{\prime} \mathrm{E}$ & 61 & 9.21 & 34.49 & box core \\
\hline BA226 & Bulimina sp. & DAn-1A & Anholt & 07-11-13 & $56^{\circ} 40.130^{\prime} \mathrm{N}$ & $12^{\circ} 07.000^{\prime} \mathrm{E}$ & 61 & 9.21 & 34.49 & box core \\
\hline BA233 & Bulimina sp. & DAn-1A & Anholt & $\begin{array}{l}07-11-13 \\
\end{array}$ & $56^{\circ} 40.130^{\prime} \mathrm{N}$ & $12^{\circ} 07.000^{\prime} \mathrm{E}$ & 61 & 9.21 & 34.49 & box core \\
\hline BA234 & Bulimina sp. & DAn-1A & Anholt & 07-11-13 & $56^{\circ} 40.130^{\prime} \mathrm{N}$ & $12^{\circ} 07.000^{\prime} \mathrm{E}$ & 61 & 9.21 & 34.49 & box core \\
\hline BA253 & Ammonia T14 & DAn-1A & Anholt & 07-11-13 & $56^{\circ} 40.130^{\prime} \mathrm{N}$ & $12^{\circ} 07.000^{\prime} \mathrm{E}$ & 61 & 9.21 & 34.49 & box core \\
\hline BA254 & Elphidium S4 & DAn-1A & Anholt & 07-11-13 & $56^{\circ} 40.130^{\prime} \mathrm{N}$ & $12^{\circ} 07.000^{\prime} \mathrm{E}$ & 61 & 9.21 & 34.49 & box core \\
\hline
\end{tabular}

